\title{
Multi-objective PMU placement optimization considering the placement cost including the current channel allocation and state estimation accuracy
}

\begin{abstract}
The optimal Phasor Measurement Unit (PMU) placement problem in power systems has been considered and investigated by many researchers for accurate and fast state estimation by PMU. There is a problem that the current channel cost of the PMU affects the total placement cost. This paper proposes novel formulation in multi objective optimal PMU placement which minimizes the PMU placement cost with the current channel selection and the state estimation error. The current channel selection is represented as a decision variable in the optimization. For those trade-off objective functions, the Pareto approach by Non-dominated Sorting Genetic Algorithm II (NSGA-II) is applied in the optimization. The result of the numerical experiment in this paper demonstrates the advantage of considering the appropriate PMU current channel allocation, compared to the conventional method which ignores it, in the modified IEEE New England 39-bus test system. As a result, the proposed method obtained a better Pareto solution compared to the conventional one because of consideration for the current channel selection. The proposed PMU placement shows its advantage that it is able to reduce the total PMU placement cost while it keeps the state estimation accuracy.
\end{abstract}

Keyword: Meta-heuristics; Multi-objective optimization; Phasor measurement unit (PMU); State estimation 